\title{
Multiresidue Methods for Determination of Currently Used Pesticides in Fruits and Vegetables Using QuEChERS Technique
}

\author{
Marek Biziuk and Jolanta Stocka
}

\begin{abstract}
Applied all over the world, pesticides are some of the most common pollutants of the environment because of their stability, mobility, their consequent long-term adverse effects on living organisms in general and human health in particular. For these reasons it is essential to monitor and analyse pesticide residues in the fruits and vegetables. The analysis of food samples for the presence of pesticides causes a lot of difficulties in consideration of specificity of sample preparation based on multistage operations of purification of sample containing vestigial amount of analyte with simultaneous large amount of interferents. The extraction and determination of pesticide residues in fruit and vegetable samples are discussed, as are the techniques most commonly used in these processes. The difficulties occurring at each stage in the analytical procedure are outlined.
\end{abstract}

Index Terms-Fruits, pesticides, sample preparation, vegetables.

\section{INTRODUCTION}

Main source of pesticide residues in vegetable products is their direct application in the crops to eliminate different types of pest. Due to the low detection levels required by regulatory bodies and the complex nature of the matrices in which the target compounds are present, efficient sample preparation and trace-level detection and identification are important aspects in an analytical method. Multiresidue method development is difficult, due to the fact that compounds of different polarities, solubilities, volatilities and $\mathrm{pKa}$ values have to be simultaneously extracted and analysed. Fruit and vegetables are capable of retaining larger quantities of pesticides. Pesticides can accumulate in fruit skins. The crops most exposed to the presence of pesticides are grapes, citrus fruits and potatoes. Adsorbed pesticides can reduce the nutritious value of crops or alter their organoleptic properties. The present-day trend is to move away from persistent pesticides and to apply agents with a short decomposition time. Organochlorine pesticides have been replaced by organonitrogen and - phosphorus pesticides. Currently 520 approved active ingredients are used in nearly 2000 commercial products. These substances belong to more than 100 different classes. It is estimated that EU countries consume more than 300,000 tons of pesticides per annum for

Manuscript received March 13, 2014; revised May 25, 2014. This work was supported in part by a grant (No. 2012/05/N/ST4/02019) from the Ministry of Science and Higher Education, Poland.

The authors are with the Department of Analytical Chemistry, Faculty of Chemistry, Gdansk University of Technology, Narutowicza Str. 11/12, 80-233 Gdansk, Poland (e-mail: marbiziu@pg.gda.pl). crop protection alone [1].

\section{TeChNiques For IsOlating Pesticides From Fruit AND VEGETABLE SAMPLES}

Isolation and/or enrichment involve the transfer of analytes from the primary matrix to a secondary one with the concomitant removal of interferents and the increase of analyte concentrations to levels above the limit of determination (LOD) of the analytical technique used [2], [3]. The usual techniques for isolating pesticides from fruit and vegetable samples are: liquid-liquid extraction (LLE), pressurized liquid extraction (PLE), liquid-liquid microextraction (LLME), microwave-assisted extraction (MAE) and ultrasound-assisted extraction (UAE). Extract clean-up techniques are the most commonly employed solid-phase extraction (SPE), solid-phase microextraction (SPME), stir bar solvent extraction (SBSE) and gel permeation chromatography (GPC) [4]-[10]. Solid phase extraction (SPE) is currently the most popular extract clean-up technique [11]. Many of the published methods for pesticide determination in fresh fruits and vegetables use a combination of two or more commercially available SPE columns for clean-up in the normal-phase (NP) mode. Weak anionexchange sorbents such as primary secondary amine (PSA), aminopropyl (NH2), or diethylaminopropyl (DEA) modified silica are often used for clean-up of food samples together with strong anion-exchange sorbents (SAX, QMA). Other SPE clean-up approaches include the combination of GCB (graphitised carbon black) and PSA columns, the combination of $\mathrm{C} 18, \mathrm{GCB}$ and aminopropyl and the combination of GCB, PSA and SAX columns. A modification of SPE is dispersive solid phase extraction (dSPE). Dispersive-SPE involves the mixing of the sorbent with the extract in a mini-centrifuge tube to retain matrix interferents, but not analytes [12]. A modification of SPE, i.e. solid phase microextraction (SPME) [13], involves the adsorption of analytes on a fiber coated with a suitable solid phase that can be pushed out of a microsyringe. The analyte is then thermally desorbed and transferred to the GC injector. Depending on where the fibre is placed in relation to the sample, SPME can be divided into: direct (Direct Immersion DI - SPME) or headspace (HS-SPME). The method is not suitable for weakly volatile or thermally labile compounds as most pesticides are [14].

\section{QueChers TeChNiQue}

Approaches are being sought to develop pesticide 
determination techniques that are quick, simple, cheap, effective and safe. QuEChERS (Quick, Easy, Cheap, Effective, Rugged and Safe) [15]-[24] is one such method. QuEChERS is highly effective sample preparation technique for the pesticide multiresidue analysis in various sample matrices. This method is based on a salting-out extraction with a solvent (mainly acetonitrile) followed by a dispersive solid phase extraction (d-SPE) (see Fig. 1), and was developed by Anastassiades et al. [25]. Samples are milled in frozen state (dry ice is added) to get the best recovery. Extraction is done in acetonitrile (ACN) buffered at $\mathrm{pH}$ 5-5.5. After centrifugation, the organic phase is cleaned-up by dispersive SPE using primary secondary amine - PSA (and graphitized carbon black-GCB as necessary). Additional MgSO4 is added to remove any residual water. The PSA treated extract is acidified with formic acid to improve the stability of base-sensitive pesticides. The extract is ready for gas chromatography (GC) or liquid chromatography (LC) analysis. For samples with low water content $(<80 \%)$, water is added before the initial extraction to get a total of ca. $10 \mathrm{~mL}$ water. Quality control is performed by adding internal standard ISTD to the acetonitrile extraction step [26]. The consumption of sample and toxic solvents with the QuEChERS method is minimal. By applying QuEChERS to the determination of pesticides in fruit and vegetables, matrix effects are reduced and high recoveries of target analytes are possible. The method can be modified depending on the type of sample and the target analytes. To improve the extraction of polar base-sensitive pesticides, the method is modified by the use of buffering at $\mathrm{pH}$ ca. 5. When samples of citrus fruit are under investigation, protective wax coatings can be removed by freezing the samples for at least one hour. For samples, with a high content of carotenoids or chlorophyll, cleanup with PSA is not satisfying and there is a need to use GCB which is best in handling and effect. A more practical way to perform the dispersive SPE method is to use disposable pipet extraction (DPX) tips. These tips have a screen that contains loose sorbent material inside the pipet tip. By incorporating anhydrous magnesium sulfate $\left(\mathrm{MgSO}_{4}\right)$ and primary and secondary amine (PSA) for sorbent, these tips are referred to as QuEChERS Tips. The use of QuEChERS Tips has been reported previously and found to provide similar results to dispersive tubes. Kaewsuya et al. developed automated method of QuEChERS tips for analysis of pesticide residues in fruits and vegetables by GC-MS [27]. The QuEChERS approach is very flexible and it serves as a template for modification. Two differing standard exist with regard to the buffer type: the American standard (AOAC), which involves the use of acetate buffer, and the European standard EN 15662, which involves the use of citrate buffer. QuEChERS approach takes advantages of the wide analytical scope and high degree of selectivity and sensitivity provided by gas and liquid chromatography (GC and LC) coupled to mass spectrometry (MS) for detection. This method is simple and rapid and requires low solvent consumption, which, in the era of green chemistry, represents a significant advantage. Therefore, this technology is of great interest because of it's favorable toxicological, environment and economic aspects.

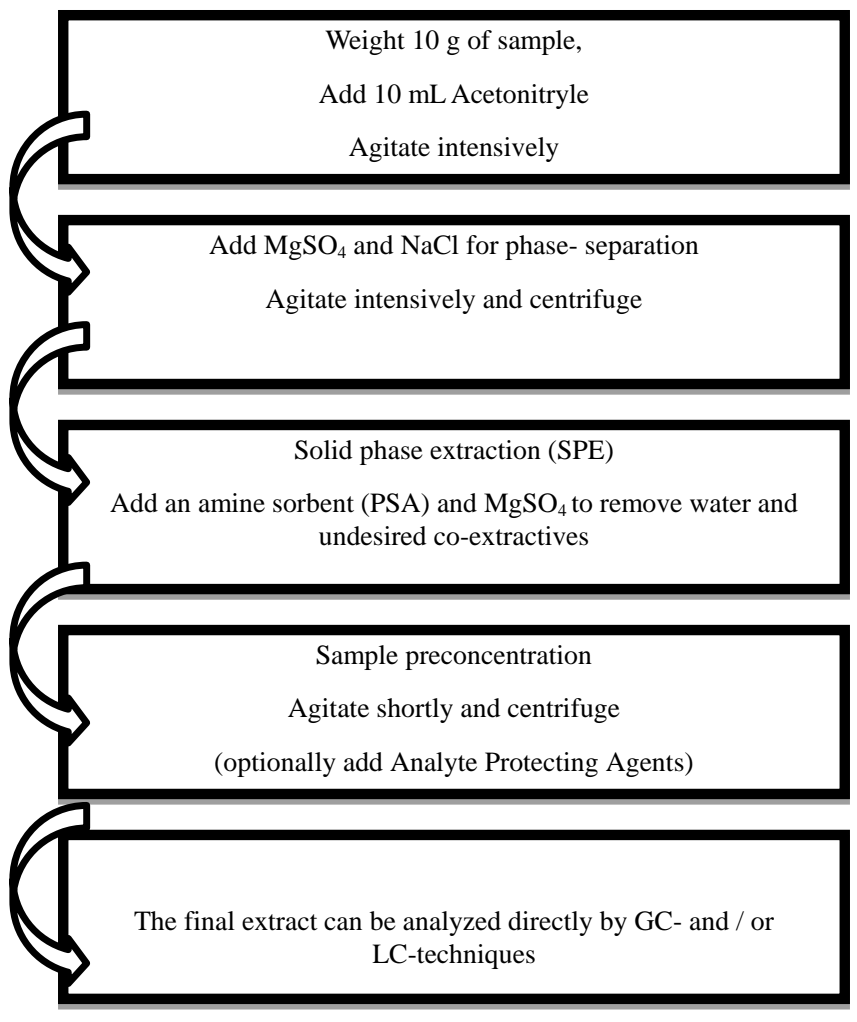

Fig. 1. Steps in the QuEChERS procedure of sample preparation for the determination of pesticide residue in fruit and vegetables

\section{FINAL DETERMINATION}

The last stage in the analytical procedure is the identification of compounds and their quantitative determination using an appropriate analytical technique.

Several multiresidue methods for determination of pesticides in fruits and vegetables using gas chromatography for separation of individual compounds, followed by detection with selective and sensitive detectors as flame photometric (FPD), pulsed flame photometric (PFPD), nitrogen-phosphorus (NPD), electron-capture detectors (ECD) or mass spectrometry detector (GC-MSD) [28]-[31].

Fenoll et al. (2007) was developed an analytical multiresidue method for the simultaneous determination of various classes of pesticides in vegetables pepper and tomato. Final determination was made by gas chromatography with nitrogen-phosphorus detection [32]. Camino-Sánchez et al. (2011) used the QuEChERS method and detection by GC-MS-MS for the quantification of 121 pesticide residues in samples of tomato, pepper, lettuce, cucumber, eggplant, zucchini, melon, watermelon and apple acquired from Spain [33].

Nowadays the use of mass spectrometry is almost mandatory. Mass spectrometry is a very sensitive and selective technique for both multiresidue determination and trace-level identification of a wide range of pesticides. MS can operate in Single Ion Monitoring (SIM) mode, which provides for greater sensitivity than the SCAN mode. MS has a wide range of application used in multi-methods for determining pesticides of different classes. Pesticides to be determined by GC should be volatile and thermally stable. During capillary GC the injector can be operated in split mode (when the stream of carrier gas is divided) or splitless 
mode (when the carrier gas stream is not divided); either mode can be used for determining pesticides in fruit and vegetable samples. The on-column injector, with which samples can be injected directly into the chromatographic column, is also used for the GC determination of pesticides. Better chromatographic peak resolution and a smaller influence of the matrix on the final result can also be achieved using two-dimensional (2D) gas chromatography (GCxGC). This uses two columns: the partially separated constituents from the first column are further separated in the second one by a different mechanism [26], [34], [35].

The selective detectors are the most common used in routine residue analysis. Unfortunately, these do not allow confirmation of the analysis results without ambiguity [36]. The detection by mass spectrometry (MS) employing quadrupole, ion trap and/or time-of-flight analysers offers simultaneously the confirmation and the quantification of numerous pesticides [37]. The time-of-flight (TOF) analyzer uses an electric field to accelerate the ions through the same potential, and then measures the time they take to reach the detector. If the particles all have the same charge, the kinetic energies will be identical, and their velocities will depend only on their masses. Lighter ions will reach the detector first. Currently, low-resolution (unit mass) MS detectors employing either single quadrupole or ion trap analysers are most routinely used in applications. Sousa et al. (2013) used gas chromatography coupled to single quadruple mass spectrometers (GC SQ/MS) for the determination of pesticide residues in Brazilian melons [38]. Unlike GC, laborious and costly derivatisation steps can be avoided in LC especially for the analysis of polar compounds. LC has been coupled to conventional detectors such as photo diode array and fluorescence detectors. However, mass spectrometry (MS) is preferred as it provides confirmatory evidence of the identity of the compound. Liquid chromatography coupled with mass spectrometry (LC-MS) or with tandem mass spectrometry (LC-MS-MS) or with time-of-flight (LC-TOF-MS) has lately become a powerful analytical technique for the identification and quantification of residues in fruits and vegetables. Thus liquid chromatography - tandem mass spectrometry (LC-MS/MS) methods based on triple quadrupole $(\mathrm{QqQ})$ analyzers are frequently used in environmental and food analysis because of the high sensitivity achieved using Selected Reaction Monitoring (SRM) acquisition mode. As a compromise between sensitivity, acceptable chromatographic peak shape, and confirmation purposes established by 2002/657/ EC directive, two SRM transitions are currently monitored [39]. Fernandez-Alba et al. (2008) described multiresidue method for determination of pesticides and their degradation products in food by advanced LC-MS. This article described the mean features of LC-MS/MS and LC-TOF-MS instruments [40]. Ferrer et al. (2005) described multiresidue method for determination of pesticides in food samples by LC-TOF-MS. This article is a valuable indicator of the potential of LC-TOF-MS to provide high-order structural information for the unequivocal identification of both targeted and non targeted pesticide residues present in fruit and vegetables [41]. Pico et al. (2007) described of fenthion metabolites in oranges by LC-QqTOF-MS and ion trap mass spectrometry (LC-IT-MS) [42].

Recently, mass spectrometry (MS/MS) has been coupled with certain advances in chromatographic technology such as Ultra Fast Liquid Chromatography (UHPLC). Carneiro et al. [43] developed multiresidue method for rapid and simultaneous determination of 128 pesticides in bananas by a modified QuEChERS procedure and UHPLC-MS/MS analysis. These techniques have made possible the development of multiresidue methodologies covering many trace contaminants. Moreover, UHPLC can reduce the analyses time and increase sensitivity. Thus, high selectivity can be achieved with minimal time. Pico et al. (2007) described ultra-high-performance liquid chromatography-quadrupole time-of-flight mass spectrometry (UHPLC-QqTOF-MS) to identify the pesticide residues present in complex pear extract. These emerging technique offering more rapid and efficient separation, as well as possibility to obtain accurate mass measurement and tandem mass spectrometry (MS/MS) [44].

\section{CONCLUSION}

Determination of pesticide residues in fruit and vegetable samples is a difficult, time-consuming and laborious analytical task. Preparation of samples for analysis is one of the most important steps in the total analytical procedure. Selection of the optimal parameters for carrying out analyte extraction and extract purification is necessary in order to obtain reliable results. However, the use of modern techniques and the use of high quality equipment enables the determination even of trace level analytes. Existing techniques are being improved and new ones developed so that different classes of pesticides can be reliably determined in a quick, simple, cheap and environmentally friendly manner.

\section{ACKNOWLEDGMENT}

The investigations were financially supported by a grant (No. 2012/05/N/ST4/02019) from the Ministry of Science and Higher Education, Poland.

\section{REFERENCES}

[1] V. Andreau and Y. Pico, "Determination of currently used pesticides in biota," Analytical and Bioanalytical Chemistry, vol. 404, pp. 2659-2681, 2012.

[2] J. J. Ramos, M. J. González, and L. Ramos, "Comparison of gas chromatography-based approaches after fast miniaturized sample preparation for the monitoring of selected pesticide classes in fruits," Journal of Chromatography A, vol. 1216, pp. 7307-7313, 2009.

[3] L. Cai, S. Gong, M. Chen, and C. Wu, "Vinyl crown ether as a novel radical crosslinked sol-gel SPME fiber for determination of organophosphorus pesticides in food samples," Analytica Chimica Acta, vol. 559, pp. 89-96, 2006.

[4] L. F. C. Melo, C. H. Collins, and I. C. S. F. Jardim, "New materials for solid-phase extraction multiclass high-performance liquid chromatographic analysis of pesticides in grapes," Journal of Chromatography A, vol. 1035, pp. 51-58, 2004.

[5] A. Sanusi, V. Guillet, and M. Montury, "Advanced method using microwaves and solid-phase microextraction coupled with gas chromatography-mass spectrometry for the determination of pyrethroid residues in strawberries," Journal of Chromatography A, vol. 1046, pp. 35-40, 2004.

[6] C. Blasco, G. Font, and Y. Pico, "Comparison of microextraction procedure to determine pesticides in oranges by liquid chromatography 
mass spectrometry," Journal of Chromatography A, vol. 970, pp. 201-212, 2002.

[7] A. Kende, Z. Csizmazia, T. Rikker, V. Angyal, and K. Torkos, "Combination of stir bar sorptive extraction-retention time locked gas chromatography-mass spectrometry and auto-mated mass spectral deconvolution for pesticide identification in fruits and vegetables," Microchemical Journal, vol. 84, pp. 63-69, 2006.

[8] A. G. Sanchez, N. R. Martos, and E. Ballesteros, "Multiresidue analysis of pesticides in olive oil by gel permeation chromatography followed by gas chromatography-tandem mass-spectrometric determination," Analytica Chimica Acta, vol. 558, pp. 53-61, 2006.

[9] A. Gelsomino, B. Petroviowi, S. Tiburtini, E. Magnani, and M. Felici, "Multiresidue analysis of pesticides in fruits and vegetables by gel permeation chromatography followed by gas chromatography with electron-capture and mass spectrometric detection," Journal of Chromatography A, vol. 782, pp. 105-122, 1997.

[10] J. J. Vreuls, R. J. J. Swen, V. P. Goudriaan, M. A. T. Kerkhoff, G. A Jongenotter, and U. A. T. Brinkman, "Automated on-line gel permeation chromatography-gas chromatography for the determination of organophosphorus pesticides in olive oil," Journal of Chromatography A, vol. 750, pp. 275-286, 1996.

[11] D. Stajnbaher and L. Zupancic-Kralj, "Multiresidue method for determination of 90 pesticides in fresh fruit and vegetables using solid phase extraction and gas chromatography-mass spectrometry," Journal of Chromatography A, vol. 1015, pp. 185-198, 2003.

[12] H. Leepipatpiboon, E. Hoh, K. Mastkovska, and S. J. Lehotay, "Effect of chlorophyll on long-term GC performance in pesticide residue analysis," in Proc. 48th FPRW, 2008.

[13] H. Reinhard, F. Sager, and O. Zoller, "Citrus juice classification by SPME-GC-MS and electronic nose measurements," LWT - Food Science and Technology, vol. 41, pp. 1906-1912, 2008.

[14] C. Blasco, G. Font, J. Manes, and Y. Pico, "Solid-phase microextraction liquid chromatography/tandem mass spectrometry to determine postharvest fungicides in fruits," Analytical Chemistry, vol. 75, pp. 3606-3615, 2003.

[15] M. Mezcua, C. Ferrer, J. F. García-Reyes, M. J. Martínez-Bueno, M. Sigrist, and A. R. Fernández-Alba, "Analyses of selected nonauthorized insecticides in peppers by gas chromatography/mass spectrometry and gas chromatography/tandem mass spectrometry," Food Chemistry, vol. 112, pp. 221-225, 2009.

[16] S. T. Lehotay, K. Son, H. Kwon, U. Koesukwiwat, W. Fu, K. Mastovska, E. Hoh, and N. Leepipatpiboon, "Comparison of QuEChERS sample preparation methods for the analysis of pesticide residues in fruits and vegetables," Journal of Chromatography A, vol. 1217, pp. 2548-2560, 2010.

[17] N. T. Dong, Y. J. Eun, L. D. Myung, and L. Gae-Ho, “A multiresidue method for the determination of 107 pesticides in cabbage and radish using QuEChERS sample preparation method and gas chromatography mass spectrometry," Food Chemistry, vol. 110, pp. 207-213, 2008.

[18] C. Lesueur, P. Knittl, M. Gartner, A. Mentler, and M. Fuerhacker, "Analysis of 140 pesticides from conventional farming foodstuff samples after extraction with the modified QuECheRS method," Food Control, vol. 19, pp. 906-914, 2008.

[19] B. Mayer-Helm, "Method development for the determination of 52 pesticides in tobacco by liquid chromatography-tandem mass spectrometry," Journal of Chromatography A, vol. 1216, pp. 8953-8959, 2009.

[20] O. Lacina, J. Urbanova, J. Poustka, and J. Hajslova, "Identification/quantification of multiple pesticide residues in food plants by ultrahigh-performance liquid chromatography-time-of-flight mass spectrometry," Journal of Chromatography A, vol. 1217, pp. 648-659, 2010

[21] B. Gilbert-López, J. F. García-Reyes and A. Molina-Díaz, "Sample treatment and determination of pesticide residues in fatty vegetables matrices: A review," Talanta, vol. 79, pp. 109-128, 2009.

[22] S. C. Cunha, J. O. Fernandes, and M. B. P. P. Oliveira, "Fast analysis of multiple pesticide residues in apple juice using dispersive liquid-liquid microextraction and multidimensional gas chromatography-mass spectrometry," Journal of Chromatography A, vol. 1216, pp. $8835-8844,2009$.

[23] A. Economou, H. Botitsi, S. Antoniou, and D. Tsipi, "Determination of multi-class pesti-cides in wines by solid-phase extraction and liquid chromatography-tandem mass spectrometry," Journal of Chromatography A, vol. 1216, pp. 5856-5867, 2009.

[24] A. Moral, M. D. Sicilia, and S. Rubio, "Determination of benzimidazolic fungicides in fruits and vegetables by supramolecular solvent based microextraction/liquid chromatography/fluorescence detection," Analytica Chimica Acta, vol. 650, pp. 207-213, 2009.
[25] M. Anastassiades, S. J. Lehotay, D. Stajnbaher, and F. J. Schenck, "Fast and easy multi-residue method employing acetonitrile extraction/partitioning and dispersive solid-phase ex-traction for the determination of pesticide residues in produce," Journal of AOAC International, vol. 86, pp. 412-31, 2003.

[26] J. Stocka, M. Tankiewicz, M. Biziuk, and J. Namieśnik, "Green aspects of techniques for the determination of currently used pesticides in environmental samples," International Journal of Molecular Science, vol. 12, pp. 7785-7805, 2001.

[27] P. Kaewsuya, W. E. Brewer, J. Wong, and S.L. Morgan, "Automated QuEChERS tips for GC/MS analysis of fruit and vegetables," Journal of Agricultural and Food Chemistry, 2013.

[28] S. C. Cunha, J. O. Fernandes, A. Alves, and M. B. P. P. Oliveira, "Fast low-pressure gas chromatography-mass spectrometry method for the determination of multiple pesticides in grapes, musts and wines," Journal of Chromatography A, vol. 1216, pp. 119-126, 2009.

[29] J. Nurmi and J. Pellinen, "Multiresidue method for the analysis of emerging contaminants in wastewater by ultra performance liquid chromatography-time-of-flight mass spectrometry," Journal of Chromatography A, vol. 1218, pp. 6712-6719, 2011.

[30] G. Dugo, G. D. Bella, M. La Torre, and M. Saitta, "Rapid GC-FPD determination of organophosphorus pesticide residues in Sicilian and Apulian olive oil," Food Control, vol. 16, pp. 435-438, 2005.

[31] T. Sibel, O. Gul, and A. Buket, "Simultaneous determination of various pesticides in fruit juices by HPLC-DAD," Food Control, vol. 16, pp. 87-72, 2005.

[32] J. Fenoll, P. Hellin, C. M. Martinez, M. Miguel, and P. Flores, "Multiresidue method for analysis of pesticides in pepper and tomato by gas chromatography with nitrogen-phosphorus detection," Food Chemistry, vol. 105, pp. 711-719, 2007.

[33] F. J. Camino-Sánchez, A. Zafra-Gómez, J. Ruiz-García, R. Bermúdez-Peinado, O. Ballesteros, A. Navalon, and J. L. Vílchez, "UNE-EN ISO/IEC 17025:2005 accredited method for the determination of 121 pesticide residues in fruits and vegetables by gas chromatography-tandem mass spectrometry," Journal of Food Composition and Analysis, vol. 24, pp. 427-440, 2011.

[34] J. Namieśnik, "Modern trends in monitoring and analysis of environmental pollutants," Polish Journal of Environmental Studies, vol. 10, pp. 127-140, 2001

[35] A. Beyer and M. Biziuk, "Methods for determining pesticides and polychlorinated bi-phenyls in food samples-problems and challenges,' Critical Review in Food Science, vol. 10, pp. 888-904, 2008.

[36] M. D. Hernando, A. Agüera, A. R. Fernández-Alba, L. Piedra, and M. Contreras, "Gas chromatographic determination of pesticides in vegetable samples by sequential positive and negative chemical ionization and tandem mass spectrometric fragmentation using an ion trap analyser," Analyst, vol. 126, pp. 46-55, 2001.

[37] J. L. M. Vidal, F. J. Arrebola, A. G. Frenich, J. M. Fernández, and M. Mateu-Sanchez, "Validation of a Gas Chromatographic-Tandem Mass Spectrometric Method for Analysis of Pesticide Residues in Six Food Commodities. Selection of a Reference Matrix for Calibration,' Chromatographia, vol. 59, pp. 321-327, 2004.

[38] J. D. S. Sousa, R. C. de Castro, G. de A. Andrade, C. G. Lima, L. K. Lima, M. A. L. Milhome, and R. F. do Nascimento, "Evaluation of an analytical methodology using QuEChERS and GC-SQ/MS for the investigation of the level of pesticide residues in Brazilian melons," Food Chemistry, vol. 141, pp. 2675-81, 2013.

[39] O. Núñeza, H. Gallart-Ayalaa, I. Ferrerb, E. Moyanoa, and M. T. Galcerana, "Strategies for the multi-residue analysis of 100 pesticides by liquid chromatography-triple quadrupole mass spectrometry," Journal of Chromatography A, vol. 1249, pp. 164-180, 2012.

[40] A. M. Fernandez-Alba and J. F. Garcia-Reyes, "Large-scale multi-residue methods for pesticides and their degradation products in food by advanced LC-MS," Trends in Analytical Chemistry, vol. 27, pp. 973-990, 2008

[41] I. Ferrer, J. F. Garcia-Reyes, and A. Fernandez-Alba, "Identification and quantitation of pesticides in vegetables by liquid chromatography time-of-flight mass spectrometry," Trends in Analytical Chemistry, vol. 24, pp. 671-682, 2005.

[42] Y. Pico, M. Farre, C. Soler, and D. Barcelo, "Confirmation of Fenthion Metabolites in Oranges by IT-MS and QqTOF-MS," Analytical Chemistry, vol. 79, pp. 9350-9363, 2007.

[43] R. P. Carneiro, F. A. S. Oliveira, F. D. Madureira, G. Silva, W. R. Souza, and R. P. Lopes, "Development and method validation for determination of 128 pesticides in bananas by modified QuEChERS and UHPLC-MS/MS analysis," Food Control, vol. 33, pp. 412-423, 2013. 
[44] Y. Pico, M. Farre, C. Soler, and D. Barcelo, "Identification of unknown pesticides in fruits using ultra-performance liquid chromatography-quadrupole time-of-flight mass spectrometry: Imazalil as a case study of quantification," Journal of Chromatography A, vol. 1176, pp. 123-134, 2007.

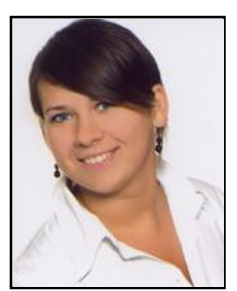

Jolanta Stocka was born in Poland. She got M. Sc. degree in biotechnology from Chemical Faculty, Gdansk University of Technology, Poland in 2009. Since 2009 she is a PhD student in the Department of Analytical Chemistry, Gdansk University of Technology. Her research areas are chemistry, environmental pollutants, and pesticides in fruits and vegetables.

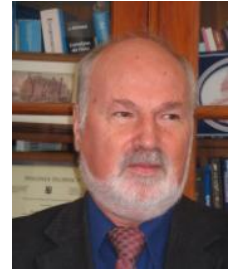

Marek Biziuk was born in Poland. He obtained his $\mathrm{PhD}$ title in 1977 from the Chemical Faculty, Gdansk University of Technology, Poland.

Since 2001 he is the professor in the Department of Analytical Chemistry, Gdansk University of Technology.

Prof. Biziuk's special scientific interests are chemistry, pesticides in the environment, organic and inorganic food and water contaminants, ecotoxicology, forensic analysis and chemometry. 
Science of Water Resources 
